\title{
The Role of Diuretics in Treatment of Aromatase Inhibitors Induced Musculoskeletal Symptoms in Women with Non Metastatic Breast Cancer
}

\author{
Alshimaa Mahmoud Alhanafy ${ }^{1 *}$, Alaa Labeeb ${ }^{2}$, Ashraf Khalil ${ }^{3}$
}

\begin{abstract}
Background: Around 50\% of women receiving Aromatase Inhibitors (AIs) develop musculoskeletal symptoms which may become severe causing interruption of treatment. Patients with AI-induced arthralgia had higher rates of joint effusions and fluid in the tendons, so use of diuretics may be helpful. Methods: This prospective phase II study was conducted in department of clinical oncology and nuclear medicine, Menoufia University Hospital, Egypt, between Jan. to Dec. 2015. Fifty Women with stage I,II and III breast cancer receiving AIs as adjuvant hormonal treatment complaining of AIs related musculoskeletal symptoms received Lasilactone ${ }^{\circledR} 50 \mathrm{mg}$ tablet; (an oral combination of Frusemide 20mg/Spironolactone $50 \mathrm{mg}$ ), every other day for 4 weeks. Patients were assessed by modified Western Ontario and McMaster Universities osteoarthritis (WOMAC) index for lower limb and the quick Disabilities of the Arm, Shoulder and Hand Score (DASH) scoring system for upper limbs, Arabic versions, at baseline and after 4 weeks of treatment. Results: The mean WOMAC pain score improved significantly $(6.0 \mathrm{v} 10 ; \mathrm{P}<0.001)$, the mean WOMAC stiffness score improved $(2.3 \mathrm{v} 3.9 ; \mathrm{P}=0.002)$, the mean WOMAC functional score improved $(8.7 \mathrm{v} 15 ; \mathrm{P}<0.001)$, the total WOMAC score improved ( 17 v 29; P $<0.001)$, also a significant difference was noticed for the quick DASH score; total score $(16 \mathrm{v} 25 ; \mathrm{P}=0.02)$ After use of diuretics for 4 weeks of treatment compared with baseline scores. Conclusions: The use of diuretics effectively reduces AI related musculoskeletal symptoms with good tolerance.
\end{abstract}

Keywords: Diuretics- aromatase inhibitors- musculoskeletal symptoms

Asian Pac J Cancer Prev, 19 (12), 3525-3531

\section{Introduction}

Currently the third-generation Aromatase Inhibitors (AIs) are an indispensable part of standard adjuvant hormonal treatment for postmenopausal women with hormone receptor positive invasive breast cancer (Burstein et al., 2016). The studies have consistently demonstrated improved disease-free survival and lower tumor recurrence when used in multiple settings: upfront in place of tamoxifen, following 2 to 3 years of tamoxifen (sequential strategy), or after completion of 5 years of tamoxifen therapy (extended strategy) (Gaillard and Stearns, 2011).

AIs have shown a more favourable overall risk-benefit profile than has tamoxifen. Benefits of the AIs include less frequent gynecologic, cerebrovascular, and thromboembolic adverse events (Thorne, 2007).

However, AIs have adverse effects, including pain, with potentially serious impact on quality of life (QOL) and treatment compliance. Fifty-seven percent of women developed pain of five different types: upper or lower limb joint pain, diffuse pain, neuropathic pain, tendon pain and mixed pain. A significant decrease in QOL was noted in the women with pain, the impact of pain on QOL depended on the type of pain experience (Laroche et al., 2017).

Many trials demonstrated that a quarter of the enrolled women discontinued initial AI therapy within 2 years specifically because of musculoskeletal symptoms, poor adherence and early discontinuation of adjuvant hormonal therapy were associated with an increased risk of poor breast cancer treatment outcomes (Hsieh et al., 2014).

Many theories have been proposed in order to explain the mechanism leading to arthralgia such as the role of estrogen deprivation and the subsequent increased sensitivity to pain stimuli following AI therapy (Robert et al., 2017). Autoimmunity and tenosynovial changes and joint effusions, several studies have recently identified characteristic radiologic changes associated with AI-induced arthralgia. In a small study that evaluated patients with severe AI-associated arthralgia, Magnetic resonant imaging (MRI) showed increased intra-articular fluid as well as enhancement and thickening of the tendon

${ }^{1}$ Department of Clinical Oncology and Nuclear Medicine, ${ }^{2}$ Department of Rheumatology, Physical Medicine and Rehabilitation, Faculty of Medicine, ${ }^{3}$ Department of Clinical Chemistry, National Liver Institute, Menoufia University, Shebin El kom, Egypt. *For Correspondence: Alshimaa_Alhanafy@yahoo.com 
sheath (Morales et al., 2008).

A retrospective analysis of women treated with adjuvant AI therapy showed that women who were on chronic diuretic treatment for heart diseases or hypertension were less likely to have symptoms of arthralgia, muscular or skeletal stiffness $(6.97 \%$ versus $15.85 \%, \mathrm{p}=0.01)$, suggesting that fluid retention within joints may play a role in AI-induced arthralgia (Xepapadakis et al., 2010).

Few forms of pain management have been established in clinical settings including the use of anti inflammatory as steroid and non steroid medication, vitamin D and glucosamine, and anti depressant medication (Gaillard and Stearns, 2011). To our knowledge, this is the first prospective study investigating the role of diuretics in management of AI-associated musculoskeletal symptoms.

\section{Materials and Methods}

\section{Patient population}

This phase II study was conducted in department of clinical oncology and nuclear medicine, Menoufia University Hospital, Egypt, between January to December. 2015. Fifty postmenopausal Women with stage I, II and III hormone receptor-positive breast cancer receiving Aromatase Inhibitors as adjuvant hormonal treatment complaining of AIs related musculoskeletal symptoms were included. This study was conducted in accordance with the Declaration of Helsinki and approved by the Committee for Ethics of Faculty of Medicine, Menoufia University, Egypt and written informed consent was obtained from each patient before enrollment.

Patients with AIs related musculoskeletal symptoms were defined by those who developed new or worsening pain during adjuvant AI therapy; Major criteria: Currently taking AI therapy, Joint pain which has developed or worsened since starting AI therapy. Minor criteria: Symmetrical joint pains, Pain in hands and/or wrists, Carpal tunnel syndrome, Decreased grip strength, Morning stiffness, Improvement in joint discomfort with use or exercise (Niravath, 2013).

Stage IV breast cancer patients receiving AIs were ineligible to avoid conflicting symptoms related to metastatic disease especially bony pains for patients with bone metastases. Patients were ineligible if they had any of the following: new pain specifically because of trauma, liver dysfunction, impairment of kidney function, creatinine clearance $<30 \mathrm{~mL} / \mathrm{min}$, a clinically significant coagulation disorder, hypersensitivity, hypotension, dehydration, concurrent use of diuretics and or any contraindication of diuretics use.

\section{Treatment Plan}

Enrolled patients continued treated with the standarddose of a third-generation AI; $1 \mathrm{mg}$ Anastrozole (Arimidex; AstraZeneca Pharmaceuticals), $25 \mathrm{mg}$ Exemestane (Aromasin; Pfizer), or $2.5 \mathrm{mg}$ Letrozole (Femara; Novartis) orally daily. Patients who had been taking a stable dose of medication for treatment of pain (eg, nonsteroidal anti-inflammatory medications, cyclooxygenase-2 inhibitors and acetaminophen), and/or bisphosphonates every 6 month at the time of enrollment were permitted to continue the medication. Lasilactone ${ }^{\circledR}$ $50 \mathrm{mg}$; (produced by: Sanofi Aventis Egypt s.a.e. Under Licence of: Sanofi Aventis/ Germany) an oral combination of Frusemide $20 \mathrm{mg} /$ Spironolactone $50 \mathrm{mg}$ in a film-coated tablet, was coadministrated every other day with breakfast for 4 weeks. Patients were also instructed to avoid taking concomitant medications with the potential to interact with Lasilactone ${ }^{\circledR}$ as described in the package insert.

\section{Assessment}

The severity and the degree of musculoskeletal symptoms were evaluated using The Western Ontario and McMaster Universities (WOMAC) index scoring system, we used the Sfax modified WOMAC Arabic validated version for lower limbs (Guermazi et al, 2004) and the Quick Disabilities of the Arm, Shoulder and Hand Score (DASH) index, Arabic validated version (Alotaibi et al., 2016) for measuring the disabilities of the arm, shoulder and hand before and at 4 weeks after initiation of diuretic therapy.

The modified WOMAC index is a self-administered composite questionnaire includes questions about pain, stiffness, and in accomplishing daily life activities. The pain subscale (P subscale) includes five questions about pain for 'walking on a flat surface' (P1), 'going up or down stairs' (P2), 'at night while in bed' (P3), 'sitting or lying' (P4), and 'standing upright' (P5). The stiffness subscale (S subscale) includes two questions about stiffness 'after first wakening in the morning' (S1) and 'after sitting, lying or resting later in the day' (S2). The physical function subscale (PF subscale) includes 9 questions about the degree of difficulty when 'rising from sitting' (PF1), 'standing' (PF2), 'bending to floor' (PF3), 'walking on a flat surface' (PF4), 'going shopping' (PF5), 'putting on socks/stockings' (PF6), 'rising from bed' (PF7), 'taking off socks/stockings' (PF8), 'sitting' (PF9), Each of the 16 questions is graded either on a five-point scale (0 to 4$)$, a total score index is calculated for each patient before and after treatment (Guermazi et al., 2004).

The Quick DASH uses 11 items to measure physical function and symptoms in musculoskeletal disorders of the upper limb, this questionnaire evaluates symptoms as well as ability to perform certain activities; 1. Open a tight or new jar. 2. Do heavy household chores. 3. Carry a shopping bag or briefcase. 4 . Wash your back. 5. Use a knife to cut food. 6. Recreational activities in which you take some force or impact through your arm, shoulder or hand. 7. During the past week, to what extent has your arm, shoulder or hand problem interfered with your normal social activities with family, friends, neighbors or groups? 8. During the past week, were you limited in your work or other regular daily activities as a result of your arm, shoulder or hand problem?. 9. Tingling (pins and needles) in your arm, shoulder or hand. 10. During the past week, how much. 11. Difficulty have you had sleeping because of the pain in your arm, shoulder or hand?. Each of the 11 questions is graded either on a five-point scale (0 to 4$)$, a total score index is calculated for each patient before and after treatment (Gummesson et al., 2006).

Complete history, full physical examination, Complete blood count, Serum electrolytes; potassium, calcium, 
sodium, magnesium and creatinine level were assessed at

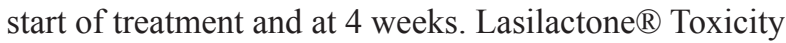
was assessed according to Common Terminology Criteria for Adverse Event v4.03 (CTCAE) Published in December 15, 2010.

Statistical Analysis: The data were collected, tabulated, and analyzed by SPSS (statistical package for social science) version 17.0 on IBM compatible computer (SPSS Inc., Chicago, IL, USA). McNemar's Chi- square test $\left(\chi^{2}\right)$ : was used to study association between paired qualitative variables. Wilcoxon Signed Rank test was used to compare two paired measurements with not normally distributed quantitative variables. Spearman correlation test was used to measure the linear correlation or dependence between two variables $\mathrm{X}$ and $\mathrm{Y}$. P values are two-sided, and confidence intervals are at the $95 \%$ level.

\section{Results}

Fifty postmenopausal women with stage I, II and III hormone receptor-positive breast cancer with Aromatase Inhibitors Induced Musculoskeletal Symptoms were evaluable with full post-treatment evaluation. Sixty two women were initially enrolled in this study but 3 women declined treatment, 4 women were irregular on diuretics because of morning diuresis and 5 women received the 4 week course but lost follow up and didn't fill post treatment evaluation at that time and presented after months of treatment but they reported general history of improvement during treatment.

The mean age was $57 \pm 7.14 \mathrm{y}$, about half of the patients in age group $55-65$ years old the mean body mass index was $34 \pm 8.23$, two thirds of the patients had BMI $>30$. Fifty two of the patients were stage III, $76 \%$ of the patients had natural menopause, $70 \%$ of the patients had no pre-existing arthralgia, $78 \%$ of the patients were recieving Letrozole, $58 \%$ of the patients received AIs 1-3 years duration, $18 \%$ of the patients had history of interrupted hormonal treatment with AIs due to these symptoms, The Median time/ months for first onset of AIs musculoskeletal symptoms is 6 months with wide range from two weeks up to 22 months. Arthralgia was the most common musculoskeletal symptom in $52 \%$ of cases, followed by stiffness $18 \%$ and myalgia $16 \%$. The upper extremity was the most common affected "single site" $30 \%$ followed by core and lower extremity $28 \%$ each. Only $24 \%$ of the patient had osteoporosis, $64 \%$ of the patients were receiving calcium and vitamin D oral supplements during the course of treatment, $17 \%$ on analgesics, and 10\% receiving bisphosphonates for osteoporosis. Table 1 and Figure 1, 2 summarize the demographic criteria.

The total WOMAC score at baseline and 4 weeks after concomitant diuretics and AI therapy significantly improved (17 v 29; P <.001). The mean WOMAC score for pain improved significantly after therapy compared with baseline $(6.0 \mathrm{v} 10 ; \mathrm{P}<.001)$, and the mean WOMAC stiffness score significantly improved ( 2.3 v $3.9 ; \mathrm{P}=.002)$. The mean WOMAC functional score improved ( $8.7 \mathrm{v} 15$; $\mathrm{P}<.001)$. Results are displayed in Table 2 and Figure 3, 4 .
Table 1. Demographic Characteristic of the Studied patients $(\mathrm{N}=50)$

\begin{tabular}{|c|c|c|}
\hline Age /year & & \\
\hline $\mathrm{X} \pm \mathrm{SD}$ & $57.76 \pm 7.14$ & \\
\hline Range & $43-79$ & \\
\hline \multicolumn{3}{|l|}{ BMI, $\mathrm{kg} / \mathrm{m}^{2}$} \\
\hline $\mathrm{X} \pm \mathrm{SD}$ & $34.16 \pm 8.23$ & \\
\hline \multicolumn{3}{|c|}{ Onset of AIs musculoskeletal symptoms/months } \\
\hline Median & 6 & \\
\hline \multirow[t]{2}{*}{ Range } & $0.5-22$ & \\
\hline & No & $\%$ \\
\hline \multicolumn{3}{|l|}{ Age groups } \\
\hline$<55$ & 18 & 36 \\
\hline $55-65$ & 26 & 52 \\
\hline$>65$ & 6 & 12 \\
\hline \multicolumn{3}{|l|}{ Menaupose range/ year } \\
\hline$<5$ & 16 & 32 \\
\hline $5-10$ & 15 & 30 \\
\hline$>10$ & 19 & 38 \\
\hline \multicolumn{3}{|l|}{ BMI } \\
\hline$<25$ & 10 & 20 \\
\hline $25-30$ & 7 & 14 \\
\hline$>30$ & 33 & 66 \\
\hline \multicolumn{3}{|l|}{ Stage } \\
\hline I & 4 & 8 \\
\hline II & 20 & 40 \\
\hline III & 26 & 52 \\
\hline \multicolumn{3}{|l|}{ Preexisting Arthralgia } \\
\hline No & 35 & 70 \\
\hline Yes & 15 & 30 \\
\hline \multicolumn{3}{|l|}{ Chemotherapy } \\
\hline No adjuvant chemotherpy & 4 & 8 \\
\hline Non Taxane based & 21 & 42 \\
\hline Taxanes Included & 25 & 50 \\
\hline \multicolumn{3}{|l|}{ Previous Tamoxifen } \\
\hline No & 37 & 74 \\
\hline Yes & 13 & 26 \\
\hline \multicolumn{3}{|l|}{ AI duration } \\
\hline$<1$ year & 12 & 24 \\
\hline 1-3 year & 29 & 58 \\
\hline$>3$ year & 9 & 18 \\
\hline \multicolumn{3}{|l|}{ Interruption of AIs } \\
\hline No & 42 & 84 \\
\hline Yes & 8 & 18 \\
\hline \multicolumn{3}{|l|}{ Osteoporosis } \\
\hline No & 38 & 76 \\
\hline Yes & 12 & 24 \\
\hline
\end{tabular}

\footnotetext{
*AI, Aromatase inhibitor, BMI, body mass index, TAM, Tamoxifen
} 
Table 2. WOMAC Score (Pain, stiffness and Functional Activity) before and after Diuretics

\begin{tabular}{|c|c|c|c|}
\hline & Baseline & 4 weeks & $\mathrm{P}$ value \\
\hline \multicolumn{4}{|l|}{ Pain score } \\
\hline $\mathrm{X} \pm \mathrm{SD}$ & $10.04 \pm 3.81$ & $6.02 \pm 4.06$ & $<0.001^{*}$ \\
\hline Range & $2-16$ & $0-15$ & \\
\hline \multicolumn{4}{|c|}{ Stiffness score } \\
\hline $\mathrm{X} \pm \mathrm{SD}$ & $3.98 \pm 2.58$ & $2.36 \pm 2.35$ & $0.002 *$ \\
\hline Range & $0-8$ & $0-8$ & \\
\hline \multicolumn{4}{|c|}{ Functional score } \\
\hline $\mathrm{X} \pm \mathrm{SD}$ & $15.18 \pm 7.04$ & $8.78 \pm 7.31$ & $<0.001^{*}$ \\
\hline Range & $0-28$ & $0-27$ & \\
\hline \multicolumn{4}{|c|}{ Womac Total score } \\
\hline $\mathrm{X} \pm \mathrm{SD}$ & $29.2 \pm 10.6$ & $17.2 \pm 12.2$ & $<0.001 *$ \\
\hline Range & $6-51$ & $0-46$ & \\
\hline
\end{tabular}

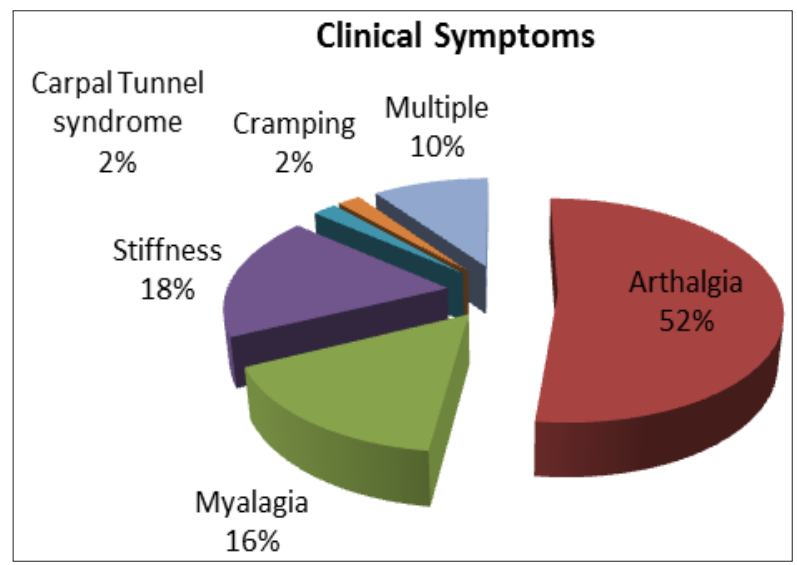

Figure 1. Clinical Symptoms of AIs Induced Musculoskeletal Symptoms

The DASH functional score improved significantly with use of diuretics with mean $29.33 \pm 23.97$ at baseline evaluation versus $19.08 \pm 22.08$ at 4 weeks, $\mathrm{p}=0.03^{*}$, also The DASH Activity score improved significantly with mean $13.50 \pm 18.53$ at baseline evaluation versus $6.25 \pm 12.69$ at 4 weeks, $\mathrm{p}=0.036^{*}$, DASH total score improved significantly with mean $25.27 \pm 20.94$ at baseline evaluation versus $16.32 \pm 18.12$ at 4 weeks, $p=0.02 *$. Two
Table 3. DASH Score (Functions, Activity, and Pain) before and after Diuretics

\begin{tabular}{lccc}
\hline & Baseline & 4 weeks & P value \\
\hline functional score & & & \\
X \pm SD & $29.33 \pm 23.97$ & $19.08 \pm 22.08$ & $0.03^{*}$ \\
Range & $0-79.17$ & $0-79.17$ & \\
Activity score & & & \\
X \pm SD & $13.50 \pm 18.53$ & $6.25 \pm 12.69$ & $0.036^{*}$ \\
Range & $0-62.5$ & $0-50$ & \\
Pain score & & & \\
X \pm SD & $25.0 \pm 25.59$ & $17.50 \pm 21.18$ & 0.19 \\
Range & $0-83.33$ & $0-66.67$ & \\
DASH total score & & & \\
X \pm SD & $25.27 \pm 20.94$ & $16.32 \pm 18.12$ & $0.02^{*}$ \\
Range & $0-75$ & $0-68.18$ & \\
\hline
\end{tabular}

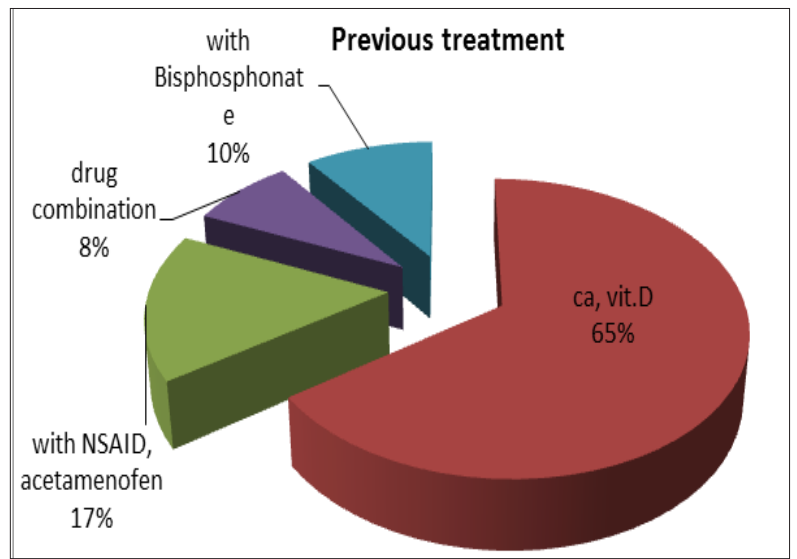

Figure 2. Previous Treatment for AIs Induced Musculoskeletal Symptoms

thirds of patients experienced at least $30 \%$ reduction in the average pain and stiffness level compared with baseline. Results are displayed in Table 3 and Figure 5.

Among the studied parameters only body mass index (BMI) showed a significant (negative) correlation with both WOMAC and DASH scores; Figure 4 and 5. All other different clinical data of the patients: the type of AIs used, age of the patients, stage of the disease, previous

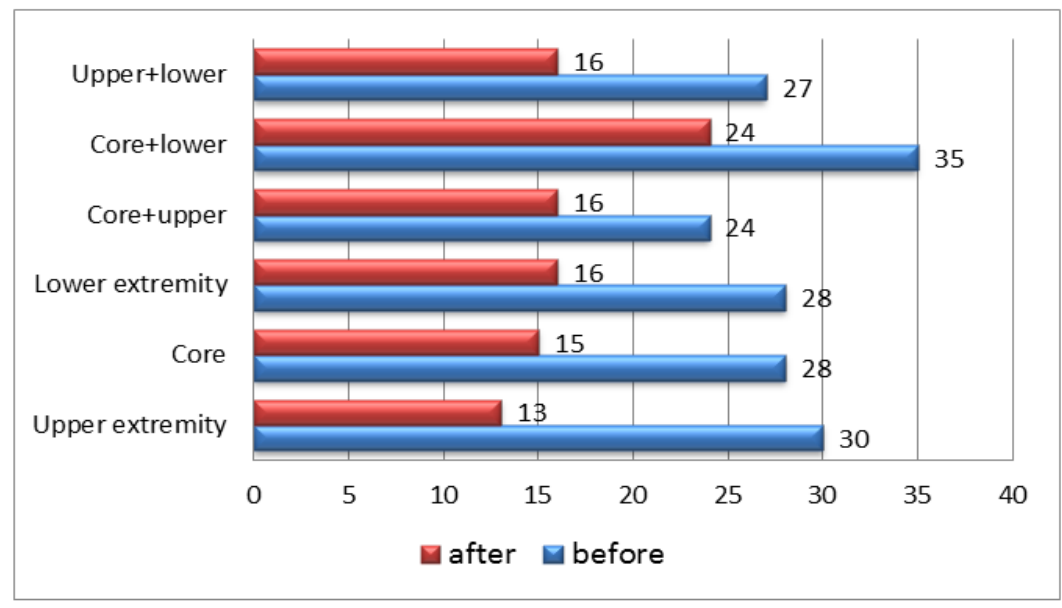

Figure 3. Improvement of Pain and Function by Anatomical Site ( $\%$ before and after Treatment)) 


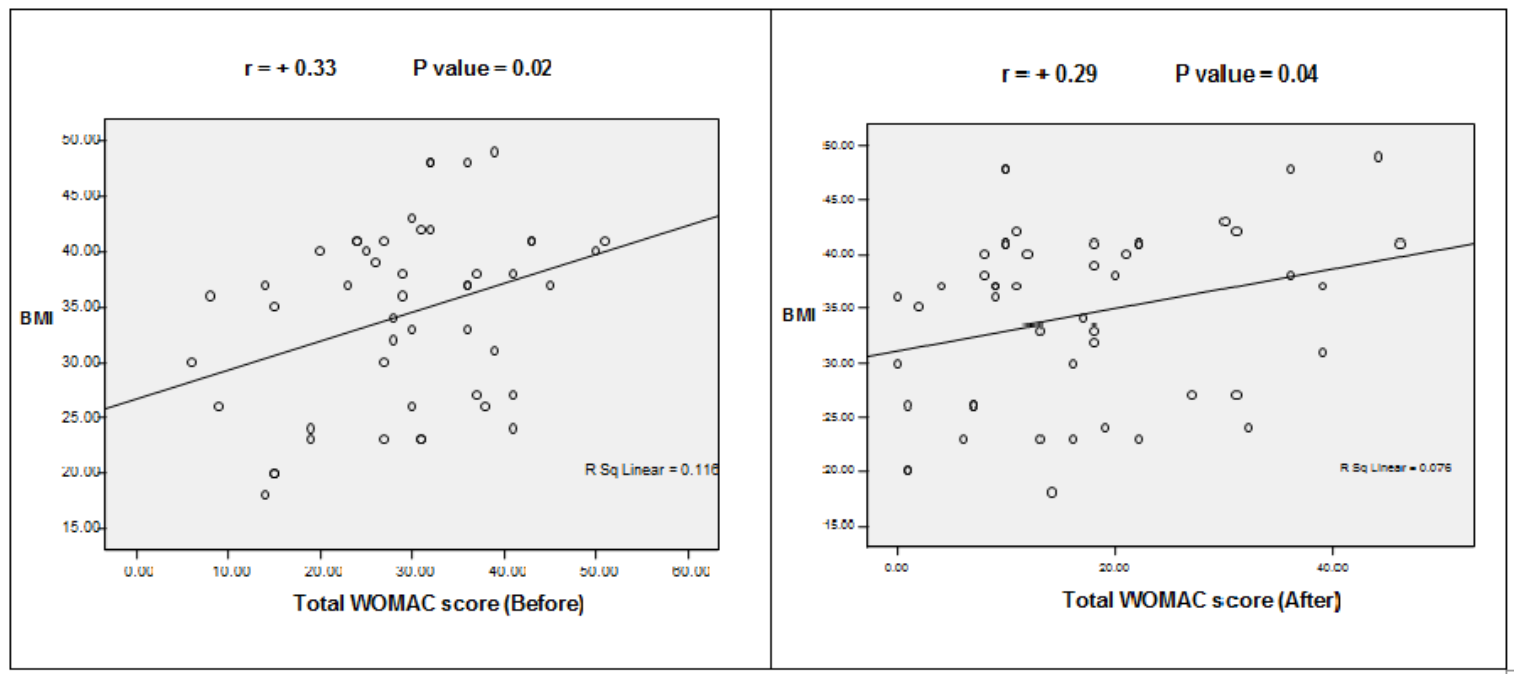

Figure 4. Total WOMAC Score (Before) and (After) Treatment and Relation to BMI

history of tamoxifen, previous history of chemotherapy especially taxanes, duration of menopause or the use of analgesics, calcium and vitamin D supplement had statistically insignificant correlations.

Regarding toxicity and tolerability; lasilactone was well tolerated; only four patients out of 59 patients who initially received the drug stopped or were irregular on diuretics because of morning dieresis which interfere with their morning work. Three patients developed grade I fatigue; which relieved by rest and 5 patients experienced grade I nausea with loss of appetite without alteration in eating habits, all continued their course of treatment.

\section{Discussion}

AIs are the standard adjuvant therapeutic care for postmenopausal women with hormone receptor positive breast cancer. Despite their beneficial effects on survival outcomes in breast cancer, AIs-induced musculoskeletal adverse effect and arthralgia are still common side effects which may lead discontinuation of the drug and decreased AIs compliance among patients (Burstein et al., 2016).

The exact patho-physiology and the underlying biological mechanisms of development of AIA is still unknown which reflect why the optimal treatment in its management has not yet been established (Nivavath, 2013). Xepapadakis et al., (2010) in a retrospective study analyzed data from 288 women receiving an AI for non-metastatic breast cancer, 42/288 Patients were receiving chronic diuretic therapy for heart diseases (Group A), while 246/288 patients had never received any diuretic medication (Group B), in Group A arthralgia was developed in $3 / 42$ patients (6.97\%) as opposed to $39 / 246$ patients in Group B (15.85\%) -p value: 0.01.

Morales et al., (2008) in a prospective study included postmenopausal patients with early breast cancer receiving either tamoxifen or an AI; an MRI of both hands and wrists were performed: AI users were more likely to develop an enhancement of the synovium, increase in intra-articular fluid, and tenosynovial changes with contrast enhancement and fluid in the tendon sheath. To our knowledge, this is the first prospective study investigating the role of diuretics in management of AI-associated arthralgia.

In this study the mean age was $57 \pm 7.14 y$, range 43 to 79 years, about half of the patients in age group $55-65$ years, this similar to results reported by Henry et al., (2011) in phase II study of duloxetine in postmenopausal

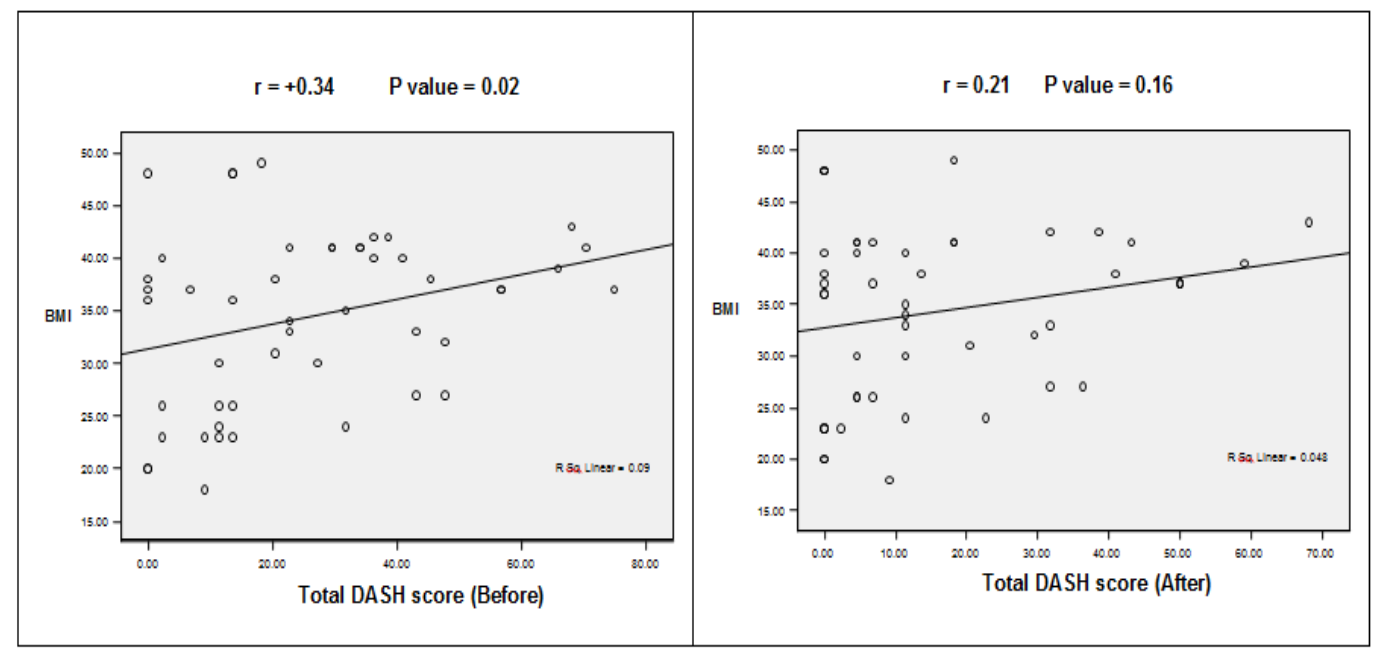

Figure 5. Total DASH Score (Before) and (After) Treatment and Relation to BMI 
women with breast cancer who developed AIs related musculoskeletal symptoms the median age was 59 years (range, 45 to 70 years) and similarly Fayed et al., (2013) reported median age 47 years, range between $55-65$ years in a cross-sectional study of the clinical and demographic risk factors associated with AI arthralgia in Egyptian breast cancer patients.

The mean body mass index, $\mathrm{kg} / \mathrm{m}^{2}$ was $34 \pm 8.23$, two thirds of the patients had BMI $>30$. Similar results reported by Fayed et al (2013); 44\% of the patients had BMI $>30$. Different results reported by Morales et al., (2008); the mean BMI in AIs groups was 22 in the European study.

Fifty two (52\%) of the patients were stage III, similarly Fayed et al (2013) reported $41 \%$ of the patients stage III, Crew et al., (2010) reported only $6 \%$ of patients with stage III in a randomized study to evaluate acupuncture for treatment of postmenopuasal women with breast cancer who had musculoskeletal pain related to AIs.

The difference in BMI as well as staging group between Egyptian and European studies is attributed to the basic differences in the patients' population of breast cancer being more advanced stage and more prevalence of obesity compared to Western patients.

In this study Thirty five out of fifty patients had no pre-existing arthralgia, similar to Fayed et al., (2013) who reported that 64 patients out of 90 patients had no previous arthritis before stating AIs.

In this study the Median time/months for first onset of AIs musculoskeletal symptoms is 6 months with wide range from two weeks up to 22 months, $24 \%$ of the studied patients received AIs less than one years, in Fayed et al., (2013) study also reported about one third of the studied patients received AIs less than one year, while Henry et al., (2011) median duration of AI therapy months 14.4 and range [2.3-46.1], Crew et al., (2010) reported Duration of AIs therapy in his study range 7-12 months.

Nine patients (18\%) had history of interrupted hormonal treatment with AIs due to associated musculoskeletal symptoms, this agrees with Canadian chart review of 50 patients found that $22 \%$ of patients discontinued adjuvant AI therapy because of toxicity, including muscle and joint symptoms (Mao et al., 2009), treatment interruption is clinically significant as more than $50 \%$ of our patients were high risk group and interruption of adjuvant treatment may jeopardize patients outcome; treatment interruption and non adherence to adjuvant hormonal therapy were found to be associated with the increasing all-cause mortality (Hsieh et al., 2014).

In this study Arthralgia was the most common musculoskeletal symptom in $52 \%$ of patients, joint stiffness in $18 \%$ of patients, Morales et al., (2008) reported osteoarthritis in $75 \%$ of the patients, $16 \%$ had Morning stiffness. The difference between the two studies is explained by the difference between the reported clinical arthralgia and the MRI radiological finding of osteoarthritis; Osteoarthritis is defined as numbers of patients with Heberden and Bouchard nodules on MRI.

In this study the upper extremity was the most common affected site in $81 \%$ of the patients; $30 \%$ of the patients had upper extremity only, $27 \%$ had upper and lower extremity and $24 \%$ had upper extremity and core. Similar results by Fayed et al., (2013); the wrist/hand (61.5\%) was the most common sites of joint pain in patients who had AI related arthralgia.

In this study 42 patients (84\%) reported improvement in their symptoms, this improvement reported early within the first week of treatment. The use of diuretic significantly reduces the pain, stiffness and improves the functional activities of the patients detected by WOMAC and DASH scores. The total WOMAC score at baseline and 4 weeks after concomitant diuretics and AI therapy significantly improved (17 v 29; P $<.001)$. DASH total score improved significantly with mean $25.27 \pm 20.94$ at baseline evaluation versus $(16.32 \pm 18.12 ; \mathrm{P}=0.02)$ at 4 weeks. Approximately two thirds of patients experienced at least $30 \%$ reduction in the average pain level compared with baseline.

Similar findings reported by Crew et al., (2010) by comparing the 6 -week WOMAC mean scores with baseline; the true acupuncture group had up to a $70 \%$ decrease in the WOMAC pain, stiffness and the functional activities scores and approximately two thirds of participants found acupuncture to be beneficial.

We could not find any specific correlation between the uses of diuretic and any of patients characteristics, only body index revealed a negative correlation with the use of 
diuretics which could be unmasked by the fact that obese women are more prone to arthritis due to the effect of weight on the joint and musculoskeletal system and other co morbidities that would be secondary to obesity as DM.

No significant toxicities, only three patients developed grade I fatigue and 5 patients experienced grade I nausea this is expected with such doses.

In conclusion the use of diuretics is an effective and safe adjuvant therapy to alleviate the AIA and musculoskeletal symptoms in breast cancer women treated with AI.

\section{Acknowledgements}

We acknowledge all patients participated in this study.

\section{References}

Alotaibi N, Aljadi S, Alrowayeh H (2016): Reliability, validity and responsiveness of the Arabic version of the disability of arm, shoulder and hand (DASH-Arabic). Disabil Rehabil, 38, 2469-78.

Burstein HJ, Lacchetti C, Griggs JJ (2016): Adjuvant endocrine therapy for women with hormone receptor-positive breast cancer: American Society of Clinical Oncology Clinical Practice Guideline Update on Ovarian Suppression Summary. J Oncol Pract, 12, 390-3.

Crew KD, Capodice,JL, Greenlee CH, et al (2010). Randomized, blinded, sham-controlled trial of acupuncture for the management of aromatase inhibitor-associated joint symptoms in women with early-stage breast cancer. J Clin Oncol, 28, 1154-60.

Fayed A, Toom MM, Alattar A, et al (2013). Arthralgia among breast cancer patients receiving adjuvant aromatase inhibitors. Life Sci J, 10, 2303-10.

Gaillard S, Stearns V (2011). Aromatase inhibitor-associated bone and musculoskeletal effects: new evidence defining etiology and strategies for management. Breast Cancer Res, 13, 205.

Guermazi M, Poiraudeau S, Yahia M, et al (2004). Translation, adaptation and validation of the Western Ontario and McMaster Universities osteoarthritis index (WOMAC) for an Arab population: the Sfax modified WOMAC. Osteoarthritis Cartilage, 12, 459-68.

Gummesson C, Ward MM, Atroshi I (2006). The shortened disabilities of the arm, shoulder and hand questionnaire (Quick DASH): validity and reliability based on responses within the full-length DASH. BMC Musculoskelet Dis, 7, 44.

Henry NL, Banerjee M, Wicha M, et al (2011). Pilot study of duloxetine for treatment of Aromatase inhibitor-associated musculoskeletal symptoms. Cancer, 117, 5469- 75.

Hsieh KP, Chen LC, Cheung KL, et al (2014). Interruption and non-adherence to long-term adjuvant hormone therapy is associated with adverse survival outcome of breast cancer women - An Asian Population-Based Study. PLoS One, 9, e87027.

Laroche F, Perrot S, Medkour T, et al (2017). Quality of life and impact of pain in women treated with aromatase inhibitors for breast cancer: A multicenter cohort study. PLoS One, 12, e0187165.

Mao JJ, Stricker C, Bruner D, et al (2009). Patterns and risk factors associated with Aromatase inhibitor related arthralgia among breast cancer survivors. Cancer, 15, 3631-9.

Morales L, Pans S, Verschueren K, et al (2008). Prospective study to assess short-term intra-articular and tenosynovial changes in the aromatase inhibitor-associated arthralgia syndrome. J Clin Oncol, 26, 3147-52.

Niravath P (2013). Aromatase inhibitor-induced arthralgia: a review. Ann Oncol, 24, 1443-9.

Robert K, Rickettb K, Greer R, et al (2017). Management of aromatase inhibitor induced musculoskeletal symptoms in postmenopausal early Breast cancer: A systematic review and meta-analysis. Crit Rev Oncol Hematol, 111, 66-80.

Thorne C (2007). Management of arthralgias associated with aromatase inhibitor therapy. Curr Oncol, 14, 11-9.

US NCI (2010). Common terminology criteria for adverse event v4.03 (CTCAE)

Xepapadakis G, Ntasiou P, Koronarchis D, et al (2010). New views on treatment of aromatase inhibitors induced arthralgia. Breast J, 19, 249-25.

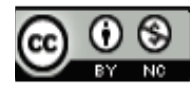

This work is licensed under a Creative Commons AttributionNon Commercial 4.0 International License. 